Trauma Berufskrankh $2005 \cdot 7$ [Suppl 2]: S334-S339 DOI 10.1007/s10039-004-0980-8

Online publiziert: 18. Februar 2005

(c) Springer Medizin Verlag 2005

F. Bonnaire $\cdot H$. Röhl

Klinik für Unfall-, Wiederherstellungs- und Handchirurgie,

Städtisches Krankenhaus Dresden-Friedrichstadt, Dresden

\title{
Epidemiologie und historischer Abriss der Behandlung
}

Verletzungen noch deren Folgen mit z. B. einer Querschnittlähmung beschrieben. Bei der Detailgenauigkeit der Kampftechnik und der Verletzungen in Homers „Ilias" muss dies verwundern. Die Ursache dafür liegt vermutlich in der Tatsache, dass eine Querschnittlähmung aussichtslos in der Behandlung war und in der Regel nur kurzfristig überlebt wurde. Der Tod als Folge der allgemeinen Komplikationen wie Dekubitalulzerationen, Blasen- und Harnwegsinfekte mit nekrotisierenden Entzündungen war gewiss, und es galt als Regel, dass man eine Lähmung nach Wirbelkörperfrakturen nur so viele Tage überleben konnte, wie es der Nummerierung der Wirbelkörper von oben nach unten (C1-LWK5) entspricht (1-24).

Diese Phase der Behandlung von Wirbelkörperbrüchen kann man durchaus als die des therapeutischen Nihilismus bezeichnen. Beschreibung von Verrenkungen eines Halswirbelkörpers mit Tetraplegie sind im Papyrus Smith [26], welches auf die Jahre 3000-2500 v. Chr. zurückgeht, vorhanden.

\section{0 v. Chr. -18. Jahrhundert: Beseitigung des Gibbus}

3000-100 v. Chr.:

Therapeutischer Nihilismus

Wer in Homers „Ilias“ oder auch der „Odyssee" nach Beschreibungen von Wirbelkörperverletzungen sucht, wird enttäuscht sein. Obwohl damals Wirbelkörperfrakturen sicher vorkamen, werden weder die
Ab 100 v. Chr. bis zum 18. Jahrhundert galt das Behandlungsbemühen in erster Linie der Beseitigung eines posttraumatischen thorakolumbalen Gibbus. Dieses Ziel wurde z. T. mit äußerst brutalen Mitteln durch Aushängen auf Repositions- und Streckbänken angestrebt. Ergebnisse über die er- reichten Repositionen und deren Komplikationen sind nicht mitgeteilt.

\section{7.-19. Jahrhundert: chirurgische Anfänge}

In der Zeit von 1700-1900 sind einige Fälle einer offenen Fragmententfernung beschrieben. Fabrizius von Hilden war der Erste, der in „hoffnungslosen Fällen“ eine offene Reposition mit einer an den Dornfortsätzen eingreifenden Zange angab. Die erste Laminektomie hat Cline [3] 1814 in London ausgeführt und gab damit Anlass für eine lange Diskussion über den Nutzen dieses Verfahrens. Sein erster Patient starb nach 9 Tagen.

Die erste operative Stabilisierung der Wirbelsäule wird Hadra [10] zugeschrieben. Er fixierte eine Luxationsfraktur des 6. und 7. Halswirbels durch einen mehrfach um die Dornfortsätze geschlungenen Silberdraht.

\section{0-1930:}

\section{Phase der Ruhigstellung}

Neben diesen noch seltenen operativen Eingriffen begann man um 1900 mit einer Ruhigstellung der Wirbelkörperfrakturen durch Bettruhe. Erst ab 1920 wurde die Bedeutung der Reposition, die Roland von Parma [25] um $1210 \mathrm{n}$. Chr. bereits beschrieben hatte, wieder zur Kenntnis genommen. Reposition, Bettruhe und anschließend Rehabilitation waren bis 1930 die Behandlungsgrundsätze. 


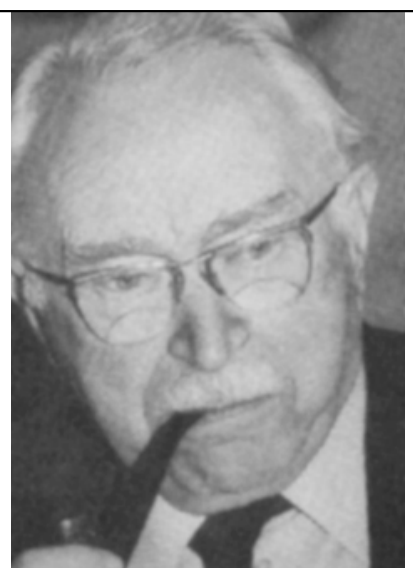

Abb. $1 \triangle$ Sir Ludwig Henry Guttmann, Begründer der umfassenden Sorge um den Querschnittpatienten

In der Folge entwickelte sich ein wissenschaftlicher Disput zwischen Magnus [21] und Lorenz Böhler [1] um die beste Behandlung der Wirbelkörperbrüche. Magnus [21] befürwortete die Behandlung von Wirbelfrakturen ohne Lähmungen nach funktionellem Schema ohne Berücksichtigung der unfallbedingten Fehlstellungen. Er ging davon aus, dass durch die unfallbedingte Fehlstellung bei der Kompression des Wirbelkörpers eine neu definierte Stabilität eintritt, die nicht durch eine Aufrichtung und damit Hinterlassung eines Defekts im Wirbelkörper behandelt werden sollte. Böhler [1] dagegen beschrieb die 3 Etappen der Behandlung mit

- Einrichtung der Fraktur,

- Ruhigstellung in Gipsverbänden und

- einer anschließenden ausgefeilten

Übungsbehandlung im Gipsmieder.

\section{0 bis heute:}

\section{chirurgische Phase dorsal}

Der eigentliche Beginn der chirurgischen Phase der Wirbelsäulenbehandlung ist auf etwa $1950 \mathrm{zu}$ datieren. Cullen [6] beschrieb 1949 eine paraspinöse Doppelplatte zur Stabilisierung von Wirbelkörpern mit dem Vorteil einer operationstechnisch einfachen und gefahrlosen Fixation. Harrington [11] stabilisierte 1962 die für die Skoliosechirurgie entwickelte Technik mit sublaminären Haken und einem Stabsystem, und Weber [31] in St. Gallen berichtete 1966 über den Einsatz von Knochenzement in Kombination mit Drahtschlingen.

Eine entscheidende Verbesserung der Stabilität wurde durch die transpediku-

\section{Zusammenfassung $\cdot$ Abstract}

Trauma Berufskrankh $2005 \cdot 7$ [Suppl 2]: S334-S339

DOI 10.1007/s10039-004-0980-8

c) Springer Medizin Verlag 2005

\section{F. Bonnaire $\cdot$ H. Röhl}

\section{Epidemiologie und historischer Abriss der Behandlung}

\section{Zusammenfassung}

Aufgrund der Häufigkeit von Frakturen der Wirbelsäule, insbesondere im thorakolumbalen Übergang, lohnt es sich, diese Region statistisch aufzuarbeiten und einen historischen Abriss der Behandlung aufzuzeigen. Vom therapeutischen Nihilismus von 3000-100 v. Chr. über Versuche, allein den Gibbus zu beseitigen, (100 v. Chr. -18. Jahrhundert), über 1. chirurgische Behandlungsversuche im 17.-19. Jahrhundert, über die kurze Phase der Ruhigstellung der Patienten mit Bettruhe von 1900-1930 und die Diskussion zwischen Böhler und Magnus wurden schließlich die bis heute verwendete chirurgische dorsale Versorgung sowie die ventralen Zugänge entwickelt. In der Versorgung von Querschnittgelähmten sind insbesondere die Arbei- ten von Guttmann zu erwähnen. Die Klassifikation nach Magerl et al. verbesserte Verständnis und Behandlungsergebnisse. Anwendung der Navigation, Wirbelkörperersatzmaterialien usw. sind weitere Neuerungen. Optimale Behandlungswege müssen sich aber in Zukunft an den Spätergebnissen messen lassen. Der Unfallchirurg sollte in seine Entscheidung für das gewählte Verfahren seine Erfahrungen und die in der Literatur berichteten kritisch einfließen lassen.

\section{Schlüsselwörter}

Wirbelsäulenfrakturen · Thorakolumbaler Übergang · Dorsale Versorgung ·

Ventraler Zugang · Querschnittlähmung

\section{Epidemiology and historical outline of treatments}

\section{Abstract}

Because spinal fractures are so common, especially at the thoracolumbar junction, we thought it worthwhile to perform a statistical workup relating to this region and to prepare a historical outline of their treatment. The development of the surgical treatments by the dorsal approach still used today and of the ventral approaches is traced from the therapeutic nihilism that held sway from 3000 to 100 B.C. through attempts directed exclusively at the correction of gibbus (100 B.C. up to the eighteenth century), through the initial attempts at surgical treatment in the seventeenth to nineteenth centuries and the brief period from 1900 to 1930 when fractures were treated with bedrest and the discussion between Boehler and Magnus. Guttmann's publications deserve a special mention in connection with the treat- ment of transverse spinal cord syndrome. The classification system devised by Magerl et al. improved our understanding of spinal syndromes and also the treatment outcomes. The use of computer-assisted navigation systems and the availability of materials for the replacement of vertebral bodies are further new developments. In the future, however, treatment methods must be measured against the long-term results. In deciding on a particular procedure the trauma surgeon must critically review his or her own experiences and those in the literature and take account of these.

\section{Keywords}

Spinal fractures - Thoracolumbar junction .

Dorsal surgery - Ventral approach .

Transverse spinal cord syndrome 
läre Fixation mit dorsaler Verplattung durch Roy-Camille u. Berteaux [29] erreicht. Eine Erweiterung dieses Prinzips einschließlich Repositionsmöglichkeit wurde mit dem Fixateur externe der Wirbelsäule von Magerl [18] 1982 angegeben. Dieses System wurde von Dick 1984 [8] modifiziert und in den uns bekannten Fixateur interne umgewandelt.

Gelenkfortsatzverschraubungen wurden von Toumey [30] 1943 und Magerl [19] in einer Verbesserung 1982angegeben.

Daniaux [7] empfahl 1982 erstmals die transpediukläre Reposition und Spongiosaauffüllung des Wirbelkörpers. Er beschrieb auch die transpedikuläre Entfernung der zerrissenen Bandscheiben und die Spongiosaauffüllung des Zwischenwirbelraums zur intrakorporellen ventralen Fusion.

\section{4 bis heute: chirurgische Techniken ventral}

Alle bisher genannten Techniken begründeten sich in erster Linie aufgrund des ungefährlichen dorsalen Zugangs zur Wirbelsäule. Ventrale Zugänge zur Wirbelsäule wurden bereits 1934 von Ito et al. [14] für die Behandlung von tuberkulösen Herden in der Wirbelsäule angegeben. Hodgson u. Stock [13] beschrieben die gesamten vorderen Zugänge zur Wirbelsäule bei Operationen der Tuberkulose. Diese beinhalteten auch die nach ihnen benannte Thorakophrenolumbotomie als maximale Freilegung und maximal invasivem Zugang zur Wirbelsäule.

Nach der Entwicklung des ventralen Zugangs zur Halswirbelsäule durch Cloward [4] und der nach ihm benannten ventralen Fusion wurden diese Zugänge zunehmend auch für die interkorporelle Fusion von Brust- und Lendenwirbelkörpern verwendet. Zusätzlich wurden später zur Sicherung der eingebrachten Späne und der erreichten Reposition auch Implantate, wie Platten oder Körbe, von ventral eingebracht.

Die großen Zugänge zur Wirbelsäule gerieten wegen ihrer Morbidität immer mehr in die Kritik, und es entwickelten sich minimalinvasive (Zwerchfellsplit), transpleurale, retroperitoneale, später auch transthorakale endoskopische Zugänge und Stabilisierungsmethoden. Ab etwa 1995 gibt es erste größere Statistiken über die erreichten Ergebnisse, die von Bühren [2] mitgeteilt wurden.

\section{Dorsalisten und Ventralisten}

Nach dem 2-Säulen-Konzept von Whitesides [32], welches dieser 1977 postulierte, ist die vordere Säule, bestehend aus den Wirbelkörpern und den Bandscheiben, in erster Linie druckbelastet, die dorsale Säule, bestehend aus den Wirbelbögen und den dorsalen Bandstrukturen, eher zugbelastet. Dieses einfache Modell hat sich für die Versorgung von Wirbelkörperfrakturen als sehr dienlich herausgestellt.

In den letzten Jahrzehnten entwickelte sich eine ausgiebige und sehr kontrovers geführte Diskussion zwischen den so genannten „Ventralisten“ und „Dorsalisten“. Diese überspitzte Bezeichnung bedeutet, dass die Operateure, die allein von dorsal stabilisieren, davon überzeugt sind, dass durch Reposition des Wirbelkörpers und die Ausschaltung der Zugkräfte eine ausreichende dauerhafte Stabilität für eine spontane Überbrückung der ventral instabilen Situation erreicht werden kann. Die „Ventralisten" hingegen sind sich sicher, dass neben der dorsalen Stabilisierung v. a. am Ort der Instabilität - der ventralen Säule eine Stabilisierung erfolgen muss.

\section{Querschnittlähmung}

Wie schon erwähnt, ist im Papyrus Smith [23] im Fall 31 eine Verrenkung an einem Halswirbel berichtet. Beschrieben wird der Lähmungszustand beider Beine und Arme, der Priapismus und die Ischuria paradoxa. Es wird gleichzeitig erläutert, dass man diese Verletzung nicht behandeln sollte. Hippokrates [12] hat die Einrichtung von Wirbelbrüchen ohne Lähmung mit Druck auf den lokalen Gibbus angegeben, im Falle der Lähmung kannte er kein Einrichtungsverfahren. Er war der Meinung, dass diese Verletzten nicht zu retten sind.

Monteggia et al. [24] warnten noch im 19. Jahrhundert vor jedem Repositionsversuch, weil es bei diesem zu einer Lähmung kommen kann. Die Tetraplegie bedeutete das Todesurteil. 3 von 100 Patienten mit Lähmungen der unteren Extremitäten überlebten wenige Wochen, nach 2 Jahren waren alle an Komplikationen verstorben.
Malgaigne [22] dagegen reponierte 1847 eine Lendenwirbelkörperfraktur durch Längszug und Lordosierung. Er berichtete auch über eine sich zurückbildende Lähmung nach diesem Manöver. Derartige Erfolge blieben jedoch extrem seltene Ausnahmen.

Ursache für die mangelnden Behandlungsmöglichkeiten war auch die schlechte Abbildbarkeit der Verletzung in der Röntgenuntersuchung. Erst 1914 war es möglich, eine Röntgenaufnahme der Wirbelsäule in ausreichender Qualität herzustellen. Der Durchbruch der operativen Behandlung der Wirbelsäulenverletzungen kam nach Einführung der CT-Diagnostik sowohl für die Klassifikation als auch für die therapeutischen Maßnahmen. Erst mit Nutzung der Röntgendiagnostik wurde bekannt, dass nur in 10-15\% der Wirbelsäulenverletzungen das Rückenmark beschädigt ist.

Auf die Diskussion zwischen Magnus und Böhler wurde bereits hingewiesen. Im Falle einer Lähmung nach Wirbelkörperfraktur wies Böhler [1] auf die Möglichkeit einer druckbedingten Durchblutungsstörung von nervalen Strukturen hin. Er forderte daher, eine Verschiebung der Wirbelsäule im Falle einer Lähmung möglichst rasch zu beheben, um den möglichen schädigenden Druck auf die ernährenden Gefäße oder das Rückenmark selbst zu beseitigen. Er beschrieb auch Rückbildungen von Teillähmungen und eine vollständige Rückbildung einer kompletten Lähmung.

Lange Zeit war es zudem eine ethische Frage, ob ein Leben mit einer Lähmung überhaupt einen Sinn habe und ob man dieses Leben verlängern dürfe. Povacz [26] beschrieb in seinem Buch über die Geschichte der Unfallchirurgie, dass noch 1942 eine Konferenz der Royal Society of Medicine über die Rehabilitation nach Gehirnverletzungen und Verletzungen der peripheren Nerven in London stattfand, und gab an, dass die Verletzungen des Rückenmarks hier überhaupt nicht zur Sprache gekommen seien.

Eine Wende in der nihilistisch-defätistischen Einstellung in der Behandlung der Wirbelkörperverletzungen hat sich mit der Vorbereitung der Invasion der Alliierten in die Normandie im Jahre 1944 eingestellt. Es wurde mit einer hohen Zahl von Rückenmarkverletzungen bei den zu erwartenden 
Kämpfen gerechnet, und bereits ab 1943 wurde eine Sonderstation für diese Verletzten geplant. Am 01.02.1944 wurde die 1. Querschnittsabteilung im Stoke Mandeville Hospital in Salesbury eröffnet. Das Leitmotiv der Klinik war die umfassende Rehabilitation der Patienten. Die Leitung dieser Abteilung wurde Herrn Dr. Ludwig Guttmann (- Abb. 1) übergeben. Er führte die intermittierende Katheterisierung durch den Arzt unter sterilen Bedingungen ein, regelmäßige Lagewechsel zur Dekubitalgeschwürprophylaxe und die Streichmassage zur Darmentleerung. Auch das Drehbett geht auf ihn zurück und führte zu einer wesentlichen Erleichterung für das Pflegepersonal. Guttmann [9] entwickelte auch eine eigene Reposition, die so genannte „Posturalreduktion“. Nach der Reposition bekämpfte er Gelenkkontrakturen durch aktives Training der Muskeln und der Gelenke. Nach Festigung der Fraktur wurde mit Stehübungen und abnehmbaren Beinschienen ein Einknicken der Knie verhindert. Guttmann unterstützte die aktive Bewegung des Verletzten durch Mobilisation im Rollstuhl und nutzte die Ergotherapie als Rehabilitationstechnik. Er führte auch Sportkämpfe unter den Gelähmten ein und erkannte den Wert des Sports für die Wiedereingliederung in ein soziales Leben. Auf seine Ideen geht die Behindertenolympiade zurück.

Er revolutionierte damit die Einstellung der Umwelt, der Angehörigen und der Verletzten bezüglich ihre zukünftigen Anforderungen und Erwartungen. Damit erreichte er - zusammen mit medizinischen Neuerungen - eine neue Akzeptanz des Krankheitsbilds und in einem hohen Prozentsatz eine Reintegration der Patienten in den Alltag [26].

Ihm gelang es, die Letalität von 3000 stationär behandelten Patienten von zuvor allgemein üblichen $90 \%$ auf $11,4 \%$ zu senken und von 2299 entlassenen immerhin 1059 in ihrer eigenen, ihren Bedürfnissen angepassten Wohnung unterzubringen. 2012 dieser Patienten wurden für industrielle, gewerbliche oder andere Arbeit wiederhergestellt. Von diesen konnte die Hälfte einer Vollbeschäftigung nachgehen.

\section{Klassifikation}

Eine umfassende Einteilung der Verletzungen wurde von Magerl et al. [20] 1994 ent-

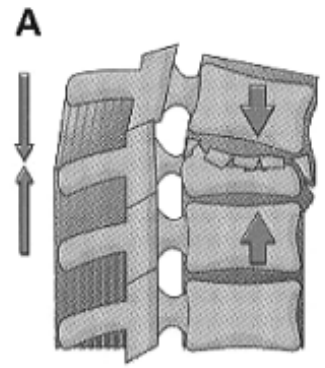

B
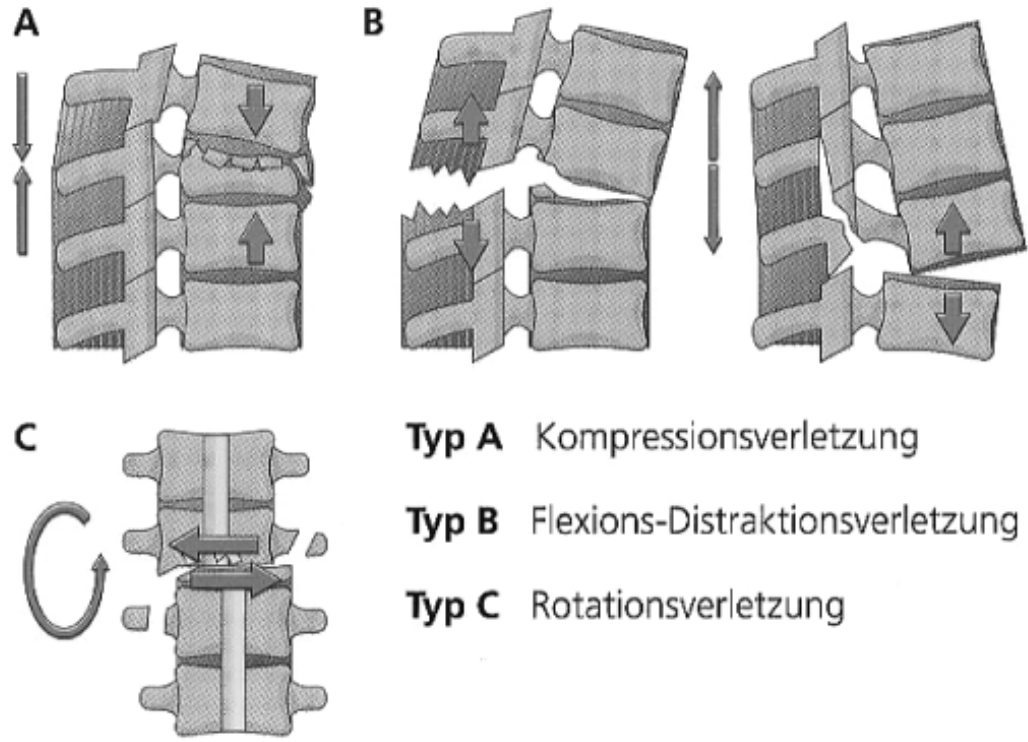

Typ A Kompressionsverletzung

Typ B Flexions-Distraktionsverletzung

Typ C Rotationsverletzung

Abb. $2 \Delta$ Klassifikation von Magerl et al. [20]

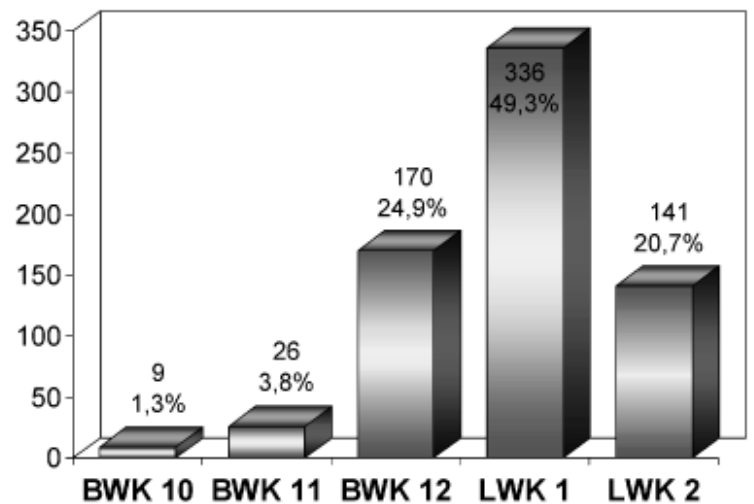

Abb. $3<$ Lokalisation und Häufigkeit der Frakturen am thorakolumbalen Übergang nach Knop et al. [15]: Dominanz des 1. LWK gegenüber seinen Nachbarwirbeln

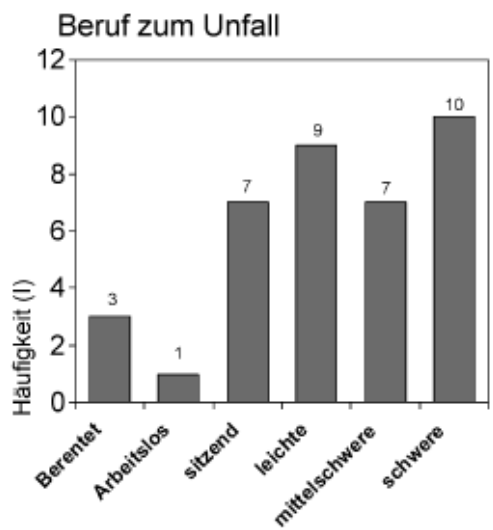

Angaben zur beruflichen Tätigkeit / körperlichen Belastung

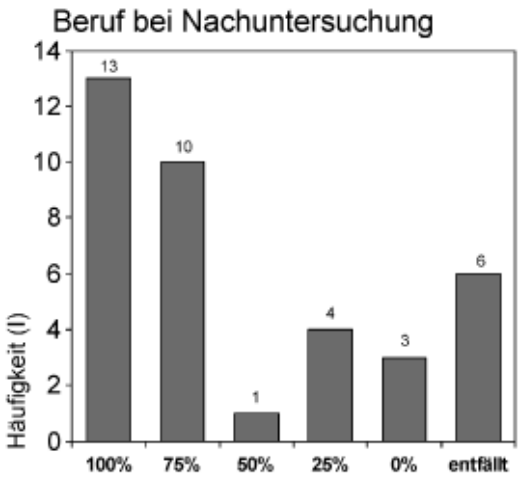

Berufliche Reintegration

Abb. 4 Berufliche Entwicklung von Patienten der MHH nach konservativer Behandlung von Wirbelfrakturen, Nachuntersuchungszeitraum 10 Jahre 
Tabelle 1

\section{Eckdaten der Studie der Arbeitsgruppe Wirbelsäule der DGU}

\begin{tabular}{|lccc}
\hline Parameter & \multicolumn{2}{l}{ Operative Behandlung } & \\
& Dorsal & Kombiniert & Ventral \\
Häufigkeit insgesamt & $448(65,7 \%)$ & $197(28,9 \%)$ & $37(5,4 \%)$ \\
\hline Alter der Patienten [Jahre] & $40,1(7-83)$ & $38,7(16-82)$ & $40.5(18-81)$ \\
\hline $\begin{array}{l}\text { Hannover-Wirbelsäulenscore } \\
\text { vor dem Unfall }\end{array}$ & $92,9(18-100)$ & $94,8(32-100)$ & $91,6(58-100)$ \\
\hline Frakturtyp A & $295(65,8 \%)$ & $112(56,9 \%)$ & $32(86,5 \%)$ \\
\hline Frakturtyp B & $93(20,8 \%)$ & $40(20,3 \%)$ & $3(8,1 \%)$ \\
\hline Frakturtyp C & $58(12,9 \%)$ & $44(22,3 \%)$ & $2(5,4 \%)$ \\
\hline Kein neurologisches Defizit & $366(81,7 \%)$ & $145(73,6 \%)$ & $27(73,0 \%)$ \\
\hline Inkomplette Querschnittläsion & $53(11,8 \%)$ & $43(21,8 \%)$ & $10(27,0 \%)$ \\
\hline Komplette Querschnittläsion & $26(5,8 \%)$ & $8(4,1 \%)$ & $0(0 \%)$ \\
\hline Keine weiteren Verletzungen & $250(55,8 \%)$ & $117(59,4 \%)$ & $26(70,3 \%)$ \\
\hline Mit Begleitverletzungen & $169(37,7 \%)$ & $68(34,5 \%)$ & $8(21,6 \%)$ \\
\hline Polytrauma & $29(6,5 \%)$ & $10(5,1 \%)$ & $3(8,1 \%)$ \\
\hline
\end{tabular}

Datne geordnet nach Lokalisation des operativem Zugangs in Abhängigkeit vom Alter, Bruchtyp, neurologischer Situation und Begleitverletzungen, modifiziert nach Knop et al. [16]

wickelt (•Abb. 2). Sie berücksichtigt erstmals die durch die bildgebende Diagnostik (CT) mögliche Einsicht in die Unfallmechanismen und regelhaft verlaufende Verletzungskombinationen der einzelnen Strukturanteile der Wirbelsäule. Im Wesentlichen sind in ihr 3 Mechanismen der Frakturentstehung beschrieben:

- Typ A umfasst die Kompressionsverletzung,

- Typ B die Flexions- und Distraktionsverletzungen und

- Typ C die Rotationsverletzungen.

Mit dieser Klassifikation wurde ein besseres Verständnis der Frakturentstehung und der notwendigen Behandlung erreicht. Sie ist an das Schema der AO-Klassifikation von Müller angelehnt und wird wegen ihrer Systematik für alle Wirbelkörper auBer $\mathrm{C}_{1}$ und $\mathrm{C}_{2}$ weltweit eingesetzt [20].

\section{Navigation}

Ein vollkommen neues Kapitel in der Behandlung von Wirbelkörperfrakturen wurde mit der chirurgischen Navigation und dem Besetzen der Pedikel unter Navigationsbedingungen aufgeschlagen. Mit dieser Art von Bildgebung und Operationssteuerung konnte die Sicherheit im The- rapieverfahren verbessert werden. $\mathrm{Zu} \mathrm{Be}-$ ginn der 1990er Jahre setzte die zunehmende Verwendung von Fremdmaterialien zum Ersatz der Wirbelkörper und der Bandscheibe ein. Ziel dieser Behandlung waren die Wiederherstellung der ursprünglichen anatomischen Säulenfunktion mit einer stabilen ventralen Komponente und die Reduktion der Entnahmemorbidität durch die bis dato am weitesten verbreitete Technik des Ersatzes mit trikortikalen autologen Knochenspänen.

\section{Osteoporotische Frakturen}

In den letzten Jahren haben sich $2 \mathrm{Me}-$ thoden zur Behandlung von osteoporotischen Wirbelkörperfrakturen in den Vordergrund gedrängt:

- die Vertebroplastie, bei welcher ein Knochenzement unter Repositionsbedingungen transpedikulär in den Wirbelkörper eingebracht wird, und

- die Kyphoplastie, welche von ihrem Grundsatz in der Anwendung etwas sicherer bezüglich Zementaustritt in Venen- und Spinalkanal erscheint. Bei dieser Technik werden Ballons transpedikulär beidseitig in den Wirbelkörper eingebracht, auf hohe Drücke aufgeblasen und der entstan- dene Hohlraum nach der angestrebten Reposition mit Knochenzement aufgefüllt.

Diese Methoden haben sich wegen der hohen Effizienz und häufig sofortigen Schmerzbefreiung sehr schnell durchgesetzt.

\section{Lokalisation und Häufigkeit von Wirbelkörperfrakturen}

Die Arbeitsgruppe „Wirbelsäule“ der DGU erfasste in einer Multicenterstudie zwischen 1993 und 1998 insgesamt etwa 1000 Wirbelkörperfrakturen am thorakolumbalen Übergang, die operativ behandelt wurden. Die Zusammenfassung der Ergebnisse wurde in einer konsekutiven Serie in „Der Unfallchirurg" von Knop et al. $[15,16,17]$, beginnend 1999, veröffentlicht.

Ursachen der Wirbelbrüche und damit der notwendigen operativen Behandlung waren meist Sturz aus größerer Höhe und Verkehrsunfälle. Am häufigsten war mit fast 50\% der 1. Lendenwirbelkörper, gefolgt vom 12. Brustwirbelkörper mit $25 \%$ und dem 2. Lendenwirbelkörper mit $20 \%$ betroffen ( $\bullet$ Abb. 3). Die Frakturtypen, die am häufigsten einer operativen Behandlung bedurften, fielen in die Kategorie der Typ-A3-Verletzung, der Kompressionsberstungsbrüche, gefolgt von den Flexions-/ Distraktionsverletzungen sowie den Kompressions-/Rotationsverletzungen ( $\bullet \mathbf{T a}$ belle 1). In dieser umfassenden Arbeit wurde eine eindeutige Korrelation der neurologischen Ausfälle zum Frakturtyp mit einer Zunahme von A-C nachgewiesen.

Bezüglich der operativen Behandlung wurden insgesamt $65,7 \%$ allein von dorsal, $28,9 \%$ kombiniert dorsoventral und nur $5,4 \%$ alleine von ventral stabilisiert. Diese Zahlen mögen eine Übersicht dafür geben, wie in spezialisierten Traumazentren in Deutschland in diesem Zeitraum die operative Behandlung in Abhängigkeit von der Verletzung, Begleitverletzungen, Alter und neurologischen Begleiterscheinungen durchgeführt wurde (•Tabelle 1$)$.

Im Rahmen der Nachuntersuchungen konnte festgestellt werden, dass das vorbestehende Aktivitätsniveau nach dem Unfall und der operativen Versorgung nur selten wieder erreicht wurde. 
Eine 10 Jahre ältere Untersuchung konnte aus dem Krankengut der Universitätsklinik Hannover nach konservativer Behandlung bezüglich der beruflichen Rehabilitation nachweisen, dass auch mit konservativer Therapie in den meisten Fällen eine gute berufliche Reintegration gelingen kann. Immerhin konnten 13 Patienten von 37, die eine stehende Tätigkeit verrichten, voll rehabilitiert werden. Allerdings waren 8 von 37 Patienten beruflich nicht mehr integrierbar (• Abb. 4) [27].

\section{Fazit}

Es gab und gibt nicht nur einen „goldenen Weg" der Behandlung von Wirbelsäulenverletzungen. Optimale Behandlungswege müssen sich in Zukunft an den Spätergebnissen messen lassen. Es wird sich erst zeigen, ob synthetische Materialien sich auf Dauer bewähren oder eine potenzielle Gefahr für den Körper darstellen. Der Unfallchirurg sollte in seine Entscheidung für das gewählte Verfahren seine Erfahrungen einfließen lassen und die Literatur kritisch betrachten und dem Patienten eine solide Behandlungsofferte anbieten können.

\section{Korrespondierender Autor}

\section{Prof. Dr. F. Bonnaire}

Klinik für Unfall-, Wiederherstellungsund Handchirurgie, Städtisches Krankenhaus Dresden-Friedrichstadt, 01067 Dresden

E-Mail: bonnaire-fe@khdf.de

Interessenkonflikt: Keine Angaben

\section{Literatur}

1. Böhler $L$ (1932) Behandlung der Wirbelbrüche. Langenbecks Arch Chir 173: 843-847

2. Bühren V (2001) Verletzungen der Brust- und Lendenwirbelsäule. Chirurg 72: 865-879

3. Cline H (1814) In: Gurlt E (1898) Geschichte der Chirurgie. Hirschwald, Berlin

4. Cloward R (1958) The anterior approach for removal of ruptured discs. J Neurosurg 15: 602-617

5. Cloward R (1961) Treatment of acute fractures and fracture dislocations of the cervical spine by vertebral-body fusion. J Neurosurg 18: 201-209

6. Cullen CH (1949) Fracture dislocation of the lumbar spine. J Bone Joint Surg Br 31: 141

7. Daniaux H (1982) Technik und erste Ergebnisse der transpedikulären Spongiosaplastik bei Komressionsbrüchen der Lendenwirbelsäule. Acta Chir Austriaca 43: 79
8. DickW (1984) [Internal fixation of the thoracic and lumbar vertebrae]. Probl Chir Orthop 28: 1-125

9. Guttmann L (1978) Total responsibility of the surgeon in the management of traumatic spinal paraplegics and tetraplegics. Paraplegia 15: 285-292

10. Hadra BE (1891) Wiring of the vertebrae as a means of immobilisation in fracture and Pott's disease. Med Times Register 22: 423

11. Harrington P (1962) Treatment of scoliosis. Correction and internal fixation by spine instrumentation. J Bone Joint Surg Am 44: 591-610

12. Hippokrates VI.106 S121-133 Kapitel 41-48

13. Hodgson AR, Stock FE (1956) Anterior spine fusion. A preliminary communication on the radical treatment of Pott's disease and Pott's paraplegia. $\mathrm{Br}$ J Surg 44: 266

14. Ito H, Tsuchiya J, Asami G (1934) A new radical operation for Pott's disease. J Bone Joint Surg Am 16: 499

15. Knop C, Blauth M, Bühren V et al. (1999) Operative Behandlung von Verletzungen des thorakolumbalen Übergangs; Teil 1: Epidemiologie. Unfallchirurg 102: 924-935

16. Knop C, Blauth M, Bühren V et al. (2000) Operative Behandlung von Verletzungen des thorakolumbalen Überganges; Teil 2: Operation und röntgenologische Befunde. Unfallchirurg 103: 1032-1047

17. Knop C, Blauth M, Bühren V et al. (2001) Operative Behandlung von Verletzungen des thorakolumbalen Übergangs; Teil 3: Nachuntersuchung. Unfallchirurg 104: 583-600

18. Magerl F (1982) External skeletal fixation of the lower thoracic and the lumbar spine. In: Uhthoff HK (ed) Current concepts of external fixation of fractures. Springer, Berlin Heidelberg New York, S 353-366

19. Magerl F (1986) Stable posterior fusion of the atlas and axis by transarticular screw fixation. In: Kehr P, Weidner A (eds) Cervical spine I. Springer, Berlin Heidelberg New York, S 322-327

20. Magerl F, Aebi M, Gertzbein SD et al. (1994) A comprehensive classification of thoracic and lumbar injuries. Eur Spine 3: 184-201

21. Magnus G (1930) Die Behandlung und Begutachtung des Wirbelbruches. Arch Orthop Unfallchir 29: 277

22. Malgaigne $F(1847)$ Traité des fractures et des luxations. Baillière, Paris

23. Meyerhof M (1931) Über den „Papyrus Edwin Smith", das älteste Chirurgiebuch der Welt. Dtsch Z Chir 231: 1931

24. Monteggia GB (1857) Istituzioni chirurgiche, vol 12, ed 3. Oliva, Milano

25. Parma R (1210) Zitiert nach Tscherne $H$, Blauth B (Hrsg) (1998) Wirbelsäule. Springer, Berlin Heidelberg New York, nach Carbonelli, in Bennet G (1984) History. In: Howorth MB, Petrie JG (eds) Injuries of the spine. Williams \& Wilkins, Baltimore, pp 1-59

26. Povacz F (2000) Geschichte der Unfallchirurgie. Springer, Berlin Heidelberg New York

27. Reinhold M, Knop C, Lange U et al. (2003) Nichtoperative Behandlung von Verletzungen der thorakolumbalen Wirbelsäule; klinische Spätergebnisse nach 16 Jahren. Unfallchirurg 106: 566-576

28. Rothe G (2002) Meldepflichtige Unfälle mit Frakturen im WS-Bereich. Hauptverband der gewerblichen Berufsgenossenschaften (HVBG), St. Augustin

29. Roy-Camille R, Saillant G, Berteaux D (1976) Osteosynthesis of thoraco-lumbar spine fractures with metall plates screwed through the vertebral pedicules. Reconstr Surg Traumatol 15: 2-16
30. Toumey JW (1943) Internal fixation in fusion of the lumbarsacral joints. Lahey Clin Bull 3: 188

31. Weber BG (1966) Early surgical treatment of traumatic paraplegia. Praxis 55: 710-713

32. Whitesides T (1977) Traumatic kyphosis of the thoracolumbar spine. Clin Orthop 128: 78-92 